\title{
The risk of the collapse of public health centres under the current system to prevent the spread of COVID-19
}

\author{
Ken Inoue $^{1} \oplus$, Yoshiyuki Ohira ${ }^{2,3}{ }^{\oplus}$, Haruo Takeshita $^{4} \oplus$ \\ ${ }^{1}$ Kochi University, Kochi, Japan \\ ${ }^{2}$ International University of Health and Welfare, School of Medicine, Chiba, Japan \\ ${ }^{3}$ Chiba University Hospital, Chiba, Japan \\ ${ }^{4}$ Shimane University, Shimane, Japan
}

COVID-19, which originated in the city of Wuhan, Hubei Province in China in December 2019, is spreading worldwide. Most of the recent media coverage in Japan has dealt with COVID-19. In its system to respond to COVID-19, Japan has criteria for determining suspected cases of COVID-19. If an individual might have COVID-19 based on those criteria, then government bodies in the form of public health centres or counselling centres (associated with public health centres) are contacted, and personnel at the public health centre or counselling centre "determine whether the individual needs to be seen by a COVID-19 centre" [1]. In addition, public health centres are responding to COVID-19 by "providing phone consultations to address COVID-19 concerns", "following up with patients with mild symptoms or individuals who might be infected", "investigating close contacts (which includes identifying clusters)", "investigating and examining routes of infection", "transporting specimens depending on the situation", and "looking for hospitals that will admit patients with COVID-19" [1, 2]. Public health personnel are performing routine tasks in addition to the aforementioned efforts to deal with COVID-19. Studies have reported that some of the workers performing over 80 hours of overtime a month (the "death from overwork limit") are in charge of the response to COVID-19 and that public health centre personnel are working throughout the crisis with almost no time off $[2,3]$.

Nationwide, there were 847 public health centres in Japan in 1994, but there are now 469 public health centres as a result of administrative reform, so the number of centres almost halved in about 30 years [2]. There are fewer public health centre physicians and public health nurses than in the past. Under Japan's current system to prevent the spread of COVID-19, public health centres are the frontline of the multifaceted response to the crisis, but the response by public health centres will falter due to the excessive workload and the dearth of personnel if nothing is done. Reports have described the "closure of emergency rooms" and "the potential risk of the medical system collapsing" [4, 5]. If such circumstances develop, then public health centres may be spending more time looking for hospitals that will see patients with COVID-19 in addition to their current tasks. Public health centres are "temporarily bringing retired public health nurses back in" and "extensively using ICT" [1], but these steps have not enhanced the ability of personnel to deal with the crisis nor have they reduced mental exhaustion suffered by personnel. Manpower needs to be increased, public health centre personnel need appropriate rest, and mental health care is urgently needed. Avoiding the potential collapse of public health centres will help correspondence during long-periods with COVID-19.

\section{REFERENCES}

1. livedoor NEWS. https://news.livedoor.com/article/detail/18165251/. (cited 2020 May).

2. Nikkansports. https://www.nikkansports.com/general/nikkan/ news/202004250000553.html. (cited 2020 May).

3. YAHOO!JAPAN news. https://news.yahoo.co.jp/articles/ 3fae27a31266788ada0097a49a198bbfaa575496. (cited 2020 April).

4. Asahi newspaper DIGITAL. https://www.asahi.com/articles/ASN4V6WFCN4QULOB003.html. (cited 2020 April).

5. YAHOO!JAPAN news. https://news.yahoo.co.jp/articles/1d774849f702295585aa449e0e5f7c54e6b659ca. (cited 2020 April). 